\title{
Design method of multiuser MIMO system using large scale transmit array with low computational cost based on subarray division
}

\author{
Tetsuki Taniguchi ${ }^{1 \mathrm{a})}$ and Yoshio Karasawa \\ ${ }^{1}$ Advanced Wireless and Communivation Research Center, The University of \\ Electro-Communications, Chofugaoka, Chofu, Tokyo 182-8585, Japan \\ a)taniguch@ee.uec.ac.jp
}

\begin{abstract}
This paper proposes an efficient design method of multiuser multiple input multiple output (MIMO) downlink system assuming use of a large array antenna in transmitter side. To reduce the heavy computational load required for transmit weight design, the large antenna is first divided into several subarrays, and block diagonalization method is applied to each of them. Then subarrays are again synthesized to a large array with the original size based on the concept of the maximum ratio combining (MRC), where two kinds of strategies, the transmitter first design and the receiver first approach are considered. Computer simulations show that the performance is degraded from the original method, but it can be achieved with significantly low computations so that it is utilizable even when the conventional block diagonalization is computationally impractical.
\end{abstract}

Keywords: massive MIMO, large array, multiuser, block diagonalization, subarray

Classification: Antennas and Propagation

\section{References}

[1] E. G. Larsson, O. Edfors, F. Tufvesson, and T. L. Marzetta, "Massive MIMO for next generation wireless systems," IEEE Commun. Mag., vol. 52, no. 2, pp. 186-195, Feb. 2014. DOI:10.1109/MCOM.2014.6736761

[2] Y. Wu and J. McAllister, "FPGA-based Tabu search for detection in large-scale MIMO systems," 2014 IEEE Workshop Signal Process. Syst. (SiPS 2014), Belfast, UK, Oct. 2014. DOI:10.1109/SiPS.2014.6986073

[3] C. Knievel, M. Noemm, and P. A. Hoeher, "Low-complexity receiver for largeMIMO space-time coded systems," IEEE $74^{\text {th }}$ Veh. Tech. Conf. 2011 (VTC 2011-Fall), San Francisco, CA, Sept. 2011. DOI:10.1109/VETECF.2011. 6093090

[4] W. Ding, T. Lv, A. Hu, and Sixi Su, "A low-complexity vector precoding scheme for large multiuser MIMO systems," 2013 16th Int. Symp. Wireless Personal Multimedia Commun. (WPMC2013), Atlantic City, NJ, June 2013.

[5] Q. H. Spencer, C. B. Peel, A. L. Swindlehurst, and M. Haardt, "An introduction to the multi-user MIMO downlink," IEEE Commun. Mag., vol. 42, no. 10, 
pp. 60-67, Oct. 2004. DOI:10.1109/MCOM.2004.1341262

[6] U. R. O. Nickel, "Subarray configurations for digital beamforming with low sidelobes and adaptive interference suppression," IEEE 1995 Int. Radar Conf., Alexandria, VA, pp. 714-719, May 1995. DOI:10.1109/RADAR.1995.522636

[7] T. Obara, S. Suyama, J. Shen, and Y. Okumura, "Joint fixed beamforming and eigenmode precoding for super high bit rate massive MIMO systems using higher frequency bands," 2014 IEEE $25^{\text {th }}$ Int. Symp. Personal, Indoor Mobile Radio Commun. (PIMRC 2014), Washington, D.C., pp. 607-611, Sept. 2014. DOI:10.1109/PIMRC.2014.7136237

[8] T. F. Chan, "An improved algorithm for computing the singular value decomposition," ACM Trans. Math. Soft., vol. 8, no. 1, pp. 72-83, March 1982. DOI: $10.1145 / 355984.355990$

\section{Introduction}

Recently, multiuser multiple input multiple output (MIMO) communication using many antenna elements in transmitter and/or receiver sides is collecting attentions as large scale or massive MIMO [1]. In this system, in spite of high performance brought by its fulfilling resource, the complexity of data precoding and/or signal detection is significantly increased, and sometimes leads to intractable computational load. To cope with this problem, many approaches have been considered; suboptimal maximum likelihood methods based on tabu search [2] or belief propagation [3] are shown to be effective for signal detection, and utilized also in precoding using vector perturbation for a large transmit array [4]. In the conventional multiuser MIMO downlink system, linear precoding techniques are widely used because of their theoretical simplicity and low computations. This study considers the reduction of computations demanded in block diagonalization [5] which is a kind of linear precoding approach, assuming utilization of a large size array in the transmitter side and much smaller number of users than the maximum possible value. For this aim, a MIMO version of subarray based strategy (for the conventional array processing, e.g., [6]) is adopted, and to transact the uncertainty of receiver weight, two types of algorithms are considered. Combination of analog and digital beamforming based on subarrays has been suggested in [7] around the same time as the idea of this work, but this paper considers the full-digital approach.

\section{System and design}

The MIMO system considered in this study has a transmitter Tx equipped with $N_{\mathrm{t}}$ antennas which sends $L_{m}$ data streams $\left\{s_{m, \ell}(t) ; \ell=0, \cdots, L_{m}-1\right\}$ at time $t$ to receiver $\operatorname{Rx}_{m}(m=0, \cdots, M-1)$ with $N_{\mathrm{r}, m}$ antennas using weight vectors $\left\{\boldsymbol{w}_{\mathrm{t}, m, \ell} \in \mathbb{C}^{N_{\mathrm{t}}} ; \ell=0, \cdots, L_{m}-1\right\}$. Notations (Tx and $\mathrm{Rx}_{m}$ are used also for representing transmit and receive arrays). After passing MIMO channel $H_{m} \in \mathbb{C}^{N_{\mathrm{r}, m} \times N_{\mathrm{t}}}$, where $\left(n_{\mathrm{r}}, n_{\mathrm{t}}\right)$-th element $H_{m, n_{\mathrm{r}}, n_{\mathrm{t}}}$ denotes the complex gain between the $n_{\mathrm{t}}$-th antenna of Tx and the $n_{\mathrm{r}}$-th antenna of $\mathrm{Rx}_{m}$, output signal $\hat{s}_{m, \ell}(t)$ is calculated at $\mathrm{Rx}_{m}$ using weight vector $\boldsymbol{w}_{\mathrm{r}, m, \ell} \in \mathbb{C}^{N_{\mathrm{r}, m}}$. The received signal of $\mathrm{Rx}_{m}$ becomes 
$\boldsymbol{y}_{m}(t)=H_{m}\left\{\sum_{n} W_{\mathrm{t}, n} \boldsymbol{s}_{n}(t)\right\}+\boldsymbol{n}_{m}(t)$, where $W_{\mathrm{t}, m}=\left[\boldsymbol{w}_{\mathrm{t}, m, 0}, \cdots, \boldsymbol{w}_{\mathrm{t}, m, L_{m}-1}\right], \boldsymbol{s}_{m}(t)=$ $\left[s_{m, 0}(t), \cdots, s_{m, L_{m}-1}(t)\right]^{T}$, and $\boldsymbol{n}_{m}(t)$ is the additive white Gaussian noise vector generated at $\mathrm{Rx}_{m}$ (this expression of the receiver signal and the procedure of precoding is same as the conventional method in [5]). In the conventional block diagonalization, weight vectors are designed by using singular value decomposition (SVD) of MIMO matrices, but the use of a large transmit array could result in impractically heavy computations. Therefore, this study considers low computational version based on subarray processing. Here two types of algorithms with different order of transmit and receive weight design are described.

Method 1 (Transmitter First Receiver Second Approach): In the proposed methods, transmit array antenna $\mathrm{Tx}$ is divided into $S$ subarrays denoted by $\mathrm{Tx}_{0}, \cdots, \mathrm{Tx}_{S-1}$ (antenna indices of $\mathrm{Tx}_{s}$ are $\mathcal{N}_{\mathrm{t}}^{(s)}=\left\{n_{\mathrm{t}, 0}^{(s)}, \cdots, n_{\mathrm{t}}^{(s)}, N_{\mathrm{t}, s-1}\right\}$ ), where the three relations $n_{\mathrm{t}, k}^{(s)} \neq n_{\mathrm{t}, \ell}^{(s)}(k \neq \ell), \mathcal{N}_{\mathrm{t}}^{\left(s_{0}\right)} \cap \mathcal{N}_{\mathrm{t}}^{\left(s_{1}\right)}=\phi\left(s_{0} \neq s_{1}\right)$, and $\bigcup_{s} \mathcal{N}_{\mathrm{t}}^{(s)}=$ $\left\{0, \cdots, N_{\mathrm{t}}-1\right\}$ should consist. The channel between $\mathrm{Tx}_{s}$ and $\mathrm{Rx}_{m}$ is represented by $H_{m}^{(s)} \in \mathbb{C}^{N_{\mathrm{r}, m} \times N_{\mathrm{t}, s}}$.

In Method 1, after this operation, subarray weight $\boldsymbol{w}_{\mathrm{t}, m}^{(s)} \in \mathbb{C}^{N_{\mathrm{t}, s}}$ for $\mathrm{Tx}_{s}$ is designed by applying the conventional block diagonalization [5] to $\left\{H_{m}^{(s)} ; m=\right.$ $0, \cdots, M-1\}$ as the transmit weight vector corresponding to the largest stream $(\ell=0)$. Then defining subarray based virtual MIMO channel by $H_{\mathrm{v}, m}=$ $\left[H_{m}^{(0)} \boldsymbol{w}_{\mathrm{t}, m}^{(0)}, \cdots, H_{m}^{(S-1)} \boldsymbol{w}_{\mathrm{t}, m}^{(S-1)}\right] \in \mathbb{C}^{N_{\mathrm{r}, m} \times S}$, receiver weight vectors $\left\{\boldsymbol{w}_{\mathrm{r}, m, \ell} ; \ell=0, \cdots\right.$, $\left.L_{m}-1\right\}$ and transmit weight combining coefficient vector $\boldsymbol{c}_{m, \ell}=\left[c_{m, \ell, 0}, \cdots\right.$, $\left.c_{m, \ell, S-1}\right]^{T} \in \mathbb{C}^{S}$ are calculated by the maximal ratio combining (MRC) design (namely, the conventional single user SVD design concerning $H_{\mathrm{v}, m}$ ) as the left and right singular value vectors of $H_{\mathrm{v}, m}$ corresponding to the $\ell$-th largest singular value (remark that the largest one is the 0 -th). The transmit weight of the original large size is finally derived as $\boldsymbol{w}_{\mathrm{t}, m, \ell}=\left[\boldsymbol{w}_{\mathrm{t}, m}^{(0) T} c_{m, \ell, 0}, \cdots, \boldsymbol{w}_{\mathrm{t}, m}^{(S-1) T} c_{m, \ell, S-1}\right]^{T}$. The number of receiver antennas is limited by inequality $N_{\mathrm{r}} \triangleq \sum N_{\mathrm{r}, m} \leq$ $\min \left\{N_{\mathrm{t}, 0}, \cdots, N_{\mathrm{t}, S-1}\right\}$ on the ground of the relation to the degrees of freedom (this inequality is introduced from the fact each subarray should be able to transact $M$ users).

Method 2 (Receiver First Transmitter Second Approach): In Method 2, receiver weights $\left\{\boldsymbol{w}_{\mathrm{r}, m, \ell}\right\}$ are first designed by using the conventional block diagonalization [5] of $\left\{H_{m} ; m=0, \cdots, M-1\right\}$ before subarray construction (the transmit weight vectors are discarded). After divided into subarrays in a similar manner as Method 1, virtual MIMO channel $H_{\mathrm{v}, m}^{(s)}=\left[H_{m}^{(s) T} \boldsymbol{w}_{\mathrm{r}, m, 0}^{*}, \cdots, H_{m}^{(s) T} \boldsymbol{w}_{\mathrm{r}, m, L_{m}-1}^{*}\right]^{T} \in$ $\mathbb{C}^{L_{m} \times N_{\mathrm{t}, s}}$ is calculated. Weight vector $\boldsymbol{w}_{\mathrm{t}, m}^{(s)}$ for transmitter subarray $\mathrm{Tx}_{s}$ is derived by applying block diagonalization to $\left\{H_{\mathrm{v}, m}^{(s)} ; m=0, \cdots, M-1\right\}$ as the transmit weight vector of the largest stream by a similar manner as Method 1. The next step is calculation of another virtual MIMO channel $H_{\mathrm{v}, m}=\left[H_{\mathrm{v}, m}^{(0)} \boldsymbol{w}_{\mathrm{t}, m}^{(0)}, \cdots\right.$, $\left.H_{\mathrm{v}, m}^{(S-1)} \boldsymbol{w}_{\mathrm{t}, m}^{(S-1)}\right] \in \mathbb{C}^{L_{m} \times S}$. And the large transmit weight with the original size is derived by the MRC design as $\boldsymbol{w}_{\mathrm{t}, m, \ell}=\left[\boldsymbol{w}_{\mathrm{t}, m}^{(0) T} c_{m, \ell, 0}, \cdots, \boldsymbol{w}_{\mathrm{t}, m}^{(S-1) T} c_{m, \ell, S-1}\right]^{T}$, where combining coefficient vector $\boldsymbol{c}_{m, \ell}$ is the right singular value vector of $H_{v, m}$ corresponding to the $\ell$-th largest singular value. The total number of data streams is upper bounded by the subarray with the minimum size, which is shown by 
$L \triangleq \sum L_{m} \leq \min \left\{N_{t, 0}, \cdots, N_{\mathrm{t}, S-1}\right\}$ because of the condition on the degrees of freedom (since one degree is consumed for one stream, $N_{\mathrm{r}}$ in the inequality in Method 1 is replaced by total number $L$ of streams). Consequently, Method 2 is a better choice if the number of the streams is smaller than the maximum possible value, but as the number of streams approaches to the maximum value $\left(L_{m}=N_{\mathrm{r}, m}\right)$, Method 1 becomes more advantageous.

\section{Simulations and discussion}

Simulations are carried out under the condition of Table I. The evaluations are based on the approximated achievable rate $C_{m}=\log _{2}\left(1+\operatorname{SINR}_{m}\right)$ (for the $m$-th user) derived from signal to interference plus noise ratio (SINR), which is calculated from the original data and the system output, and here, performance degradation from the conventional block diagonalization is observed assuming no specific application. Comparing the computational load for the SVD operation when algorithm for eigenanalysis widely used in communication is adopted, while the conventional method requires $c N_{\mathrm{t}}^{3}$ ( $c$ is a constant number) per user, that of the proposed approach is $c\left(\sum_{s} N_{\mathrm{t}, s}^{3}+S^{3}\right)\left(S^{3}\right.$ is required for the large array weight synthesis), which is only around $1 \%$ of the conventional one in the case of Table I (in case SVD routine with computations of quadratic polynomial of $N_{\mathrm{t}}$ can be utilized (e.g., [8]), cube operations in former equations are replaced by square operations, but it can still achieve about $90 \%$ reduction of computations).

Fig. 1(a) depicts the distribution functions of achievable rate. It is obvious that the performance of MIMO system by the proposed approach is degraded from the original one because of the suboptimality of the subarray processing. But it keeps quality sufficient for the use in multiuser network with a quite small computational load, which shows the proposed method is effective as a low computational method particularly when the use of the conventional version is impractical in real time applications. Since the number of streams is smaller than the maximum value,

Table I. Simulation conditions

\begin{tabular}{c|c}
\hline $\begin{array}{c}\text { Number of Receivers } \\
\text { (Number of Users) }\end{array}$ & $M=3$ \\
\hline Number of Transmit Antennas & $N_{\mathrm{t}}=100$ \\
\hline Number of Receive Antennas & $N_{\mathrm{r}, m}=2$ \\
\hline Number of Streams per User & $L_{m}=2$ \\
\hline Subarray & $S=10$ \\
Construction & $N_{\mathrm{t}, s}=10$ \\
& $\mathcal{N}_{\mathrm{t}}^{(s)}=10 s+\{0, \cdots, 9\}$ \\
& $s=0, \cdots, S-1$ \\
\hline Energy Constraint & $P_{m}=1$ \\
\hline SNR & SNR ${ }_{m}=20 \mathrm{~dB}$ \\
\hline Channel Statistics & $\begin{array}{c}\text { i.i.d. Rayleigh Fading } \\
\text { with unit variance }\end{array}$ \\
\hline
\end{tabular}




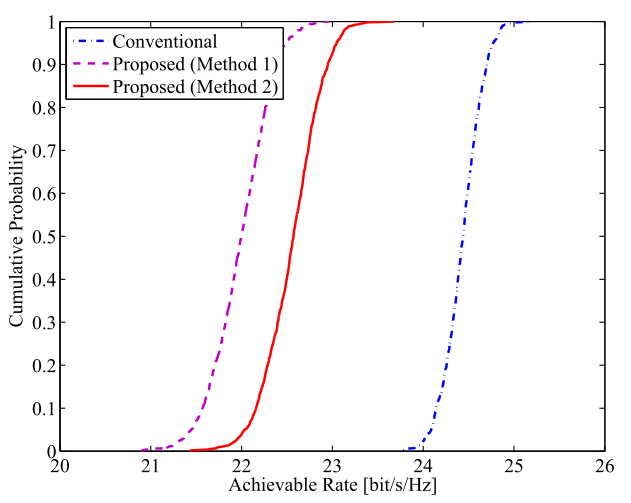

(a) Distribution functions of achievable rate.

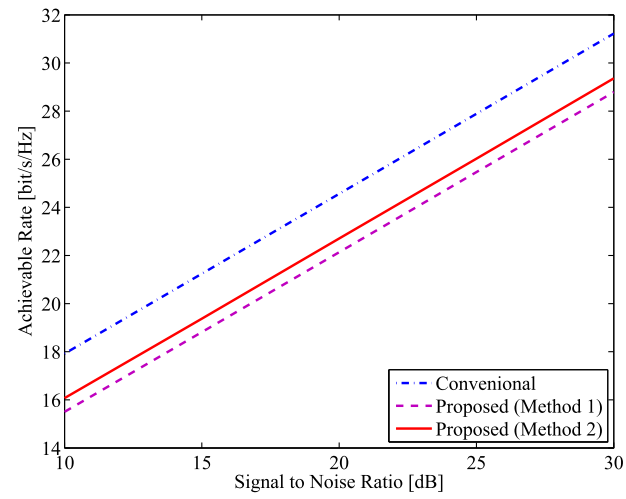

(c) Achievable rate versus SNR.

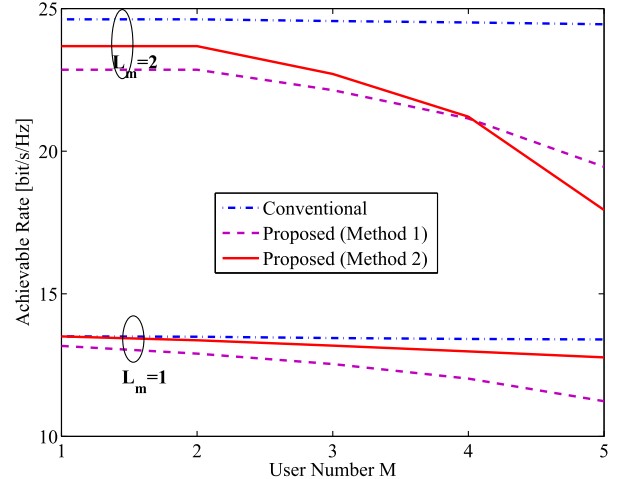

(b) Achievable rate versus Number of users $M$.

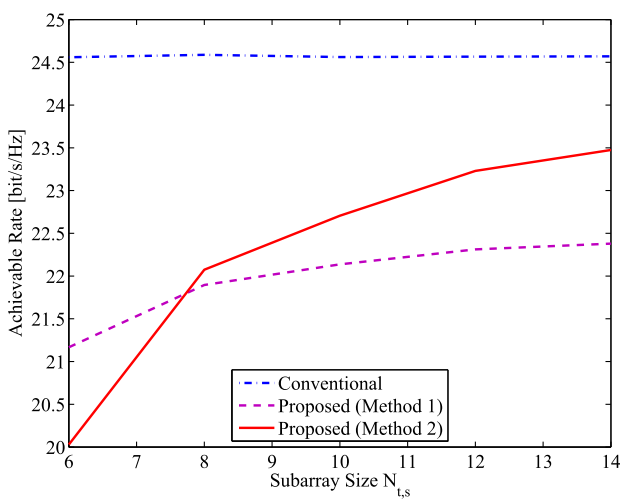

(d) Achievable rate versus subarray size $N_{\mathrm{t}, s}$.

\begin{tabular}{c|c|c|c}
\hline$N_{\mathrm{t}, s}$ & $S$ & $\begin{array}{c}\text { Subarray Size } \\
\left(N_{\mathrm{t}, 0} \sim N_{\mathrm{t}, S-3}\right)\end{array}$ & $\begin{array}{c}\text { Subarray Size } \\
\left(N_{\mathrm{t}, S-2}, N_{\mathrm{t}, S-1}\right)\end{array}$ \\
\hline \hline 6 & 16 & 6 & 8 \\
\hline 8 & 12 & 8 & 10 \\
\hline 10 & 10 & 10 & 10 \\
\hline 12 & 8 & 12 & 14 \\
\hline 14 & 7 & 14 & 15 \\
\hline
\end{tabular}

(e) Subarray grouping in (d).

Fig. 1. Performance of multiuser MIMO system designed by using proposed approach.

Method 2 which consumes less degrees of freedom has higher achievable rate under this condition.

Achievable rate versus user number $M$ curves are plotted for one and two streams in Fig. 1(b). The per-user achievable rate in the proposed method degrades from the conventional one as the increase of number of users. This is because the increment of user has larger effect on the consumption of the degrees of freedom in subarrays with much smaller size than the original large size array. Another point is that Method 1 is better than Method 2 under large total number of streams, because when $L$ is large, the disadvantage of the suboptimal receiver weight by receiver first design becomes dominant compared with the advantage of consumption of low degrees of freedom. 
The relation between SNR and achievable rate is drawn in Fig. 1(c). It can be observed that SNR does not have an influenced on the characteristics of three methods.

Fig. 1(d) shows how achievable rate is affected as the size of subarrays changes from $N_{\mathrm{t}, s}=6$ to $N_{\mathrm{t}, s}=14$. For the adjustment of the total array size $\left(N_{\mathrm{t}}=100\right)$, two subarrays could have larger size than others. The performance is improved as the size of subarrays becomes larger at the expense of the increased computational cost, but it still keeps less than $2 \%$ of the conventional method even in case of $N_{\mathrm{t}, s}=14$. As the subarray size becomes larger, benefit of Method 2 against Method 1 increases for the constant number of stream.

From the above results, it can be seen that the improvement of the design method to suppress the achievable rate reduction by the proposed method is desirable. In addition, it is remarkable that the proposed methods orient higher rate transmission by the massive array because of the limitation of subarray size, and can be switched to another approaches like sharply focused multiple beamforming when putting the priority on transaction of large number of users.

\section{Conclusion}

This paper has presented a low computational full-digital design approach of multiuser MIMO downlink system adopting a large array in transmitter side and smaller user number than maximum possible value. The massive array is first divided into several subarrays, and block diagonalization is applied to each of them, then they are again reconstructed into an array of the original size based on MRC. Computer simulations show that performance degradation from the conventional method, but it can significantly reduce the computational cost so that block diagonalization for the large array becomes practical. Future works are investigation on efficient construction of subarrays, and multistage extension of the proposed method.

\section{Acknowledgments}

This study was supported in part by the Grant-in-Aid for Scientific Research (No. 25249056) Japan Society for the Promotion of Science (JSPS). Authors will express thanks to Prof. Nobuo Nakajima, UEC, for his helpful discussion about the initial idea of this work, and to Prof. Kentaro Nishimori, Niigata University, and Dr. Riichi Kudo, NTT DoCoMo Corp. for their helpful comments. The quality of this paper has been much improved by the opinions of the editor and the anonymous reviewer. 